Service social

\title{
Hyperactivité et déficit de l'attention chez l'enfant, par Robert Dubé, Boucherville, Gaëtan Morin éditeur, 1992, 182 pages.
}

\section{Michel Pépin}

Volume 41, numéro 3, 1992

Intervenir en contexte d'autorité

URI : https://id.erudit.org/iderudit/706593ar

DOI : https://doi.org/10.7202/706593ar

Aller au sommaire du numéro

Éditeur(s)

École de service social de l'Université Laval

ISSN

1708-1734 (numérique)

Découvrir la revue

Citer ce compte rendu

Pépin, M. (1992). Compte rendu de [Hyperactivité et déficit de l'attention chez l'enfant, par Robert Dubé, Boucherville, Gaëtan Morin éditeur, 1992, 182 pages.] Service social, 41(3), 135-136. https://doi.org/10.7202/706593ar d'utilisation que vous pouvez consulter en ligne.

https://apropos.erudit.org/fr/usagers/politique-dutilisation/ 


\section{HYPERACTIVITÉ ET DÉFICIT DE L'ATTENTION CHEZ L'ENFANT}

Robert Dubé,

Boucherville, Gaëtan Morin éditeur, 1992, 182 pages.

Cet ouvrage est susceptible d'intéresser toutes les personnes qui travaillent auprès d'enfants présentant des troubles attentionnels avec ou sans hyperactivité. L'auteur est un pédiatre spécialisé dans le domaine du développement de l'enfant.

Comme son titre l'indique, l'ouvrage aborde une problématique qui préoccupe énormément toutes les personnes qui interviennent auprès d'enfants hyperactifs et qui ont à la fois le désir et la responsabilité d'aider ces enfants à cheminer dans leur vie. Comme on le sait, I'hyperactivité et les troubles attentionnels chez l'enfant ont des répercussions majeures sur son développement et entraînent souvent d'autres problèmes difficiles à régler (l'échec scolaire et toutes ses conséquences, des problèmes familiaux, des troubles de comportement, etc.). L'ouvrage du Dr Dubé apporte un éclairage récent sur ce problème qui se manifeste encore avec beaucoup d'acuité chez de nombreux enfants.

Le livre est divisé en six chapitres autour des thèmes suivants :

1. L'évolution des conceptions.

2. L'hyperactivité est-elle un syndrome spécifique?

3. L'évolution de l'hyperactivité.

4. L'évaluation.

5. La réponse aux besoins.

6. Les thérapies spécifiques.

Voici un bref aperçu de chacun des six chapitres du livre :

\section{Chapitre 1 : L'évolution des conceptions}

Le premier chapitre est centré sur une présentation des diverses conceptions et compréhensions de l'hyperactivité selon divers auteurs, tant américains qu'européens. Cette présentation ne manque pas d'intérêt, puisqu'elle montre bien la complexité de ce trouble et la difficulté à bien le définir au cours du siècle dernier. L'auteur termine ce chapitre par un rappel des critères diagnostiques de ce trouble en se référant au DSM-III-R (Diagnostic and Statistical Manual of Mental Disorders, américain) et au CIM-9 (Classification internationale des maladies, européen). II faut souligner que ce chapitre, comme les autres d'ailleurs, est accompagné d'une bibliographie exhaustive qui témoigne du soin apporté par l'auteur à bien documenter ses propos.

\section{Chapitre 2 : L'hyperactivité est-elle un syndrome spécifique?}

Ce deuxième chapitre traite divers sujets reliés à I'hyperactivité, dont les études de prévalence du trouble, les études étiologiques et les principales manifestations du trouble. L'auteur fait bien ressortir les difficultés auxquelles on se heurte lorsqu'on tente de démontrer que l'hyperactivité est un syndrome distinct ou spécifique. Le diagnostic d'hyperactivité se révèle donc extrêmement 
difficile à établir étant donné que les manifestations de ce trouble se retrouvent également sous plusieurs autres syndromes ou troubles.

\section{Chapitre 3 : L'évolution de l'hyperactivité}

À l'intérieur de ce court chapitre, l'auteur dépeint l'évolution de I'hyperactivité de l'enfance à l'âge adulte. Même si les résultats des recherches ayant porté sur l'évolution du trouble doivent être interprétés avec beaucoup de réserve, comme le souligne l'auteur, il semble que l'hyperactivité s'atténue un peu à l'adolescence et à l'âge adulte. Toutefois, chez plusieurs sujets, le trouble semble persister jusqu'à l'âge adulte.

\section{Chapitre $4:$ L'évaluation}

Ce chapitre est consacré aux divers outils permettant de diagnostiquer le trouble d'hyperactivité (l'entrevue, les questionnaires, les tests psychométriques, les mesures psychobiologiques, etc.). Chacune des méthodes d'évaluation est bien présentée avec ses avantages et ses limites. L'auteur souligne que l'efficacité des diverses méthodes d'évaluation dépend plus de la compétence de l'utilisateur que des qualités propres à la méthode d'évaluation choisie.

\section{Chapitre 5 : La réponse aux besoins}

Le professeur Dubé rappelle que l'intervention auprès des enfants hyperactifs doit tenir compte des besoins particuliers de chaque enfant plutôt que d'être centrée sur une approche théorique particulière. Il énonce donc plutôt dans ce chapitre les principes directeurs qui devraient guider l'intervention, de même que les principaux objectifs de l'intervention.

\section{Chapitre 6 : Les thérapies spécifiques}

Ce dernier chapitre aborde brièvement les thérapies comportementales, les thérapies cognitives et les thérapies basées sur la prise de médicaments (stimulants).

Le livre du Dr Dubé présente un intérêt certain pour tous les intervenants (éducateurs, parents, psychologues, travailleurs sociaux, etc.) qui sont en relation avec un ou des enfants hyperactifs ou présentant des troubles d'attention. Sans être un ouvrage de vulgarisation, le livre est à la portée de tous les professionnels, peu importe leur discipline. Il s'agit d'un livre qui se lit très rapidement (style clair, sans abus de termes techniques ou propres à une discipline). On peut donc le considérer comme un ouvrage plus pratique que théorique, cela étant dit de façon non péjorative. On y trouve d'ailleurs plusieurs éléments intéressants pour les praticiens, tels les questionnaires de Connors, une grille d'analyse des comportements et les critères diagnostiques du DSM-III-R et du CIM-9 et CIM-10. En résumé, I'auteur nous présente un ouvrage centré sur une problématique importante qu'il aborde globalement avec beaucoup d'intelligence.

Michel PÉPIN 